\title{
Exploiting Visual and Text features for Direct Marketing Learning in Time and Space Constrained Domains
}

\author{
S. Battiato ${ }^{1}$, G. M. Farinella ${ }^{1}$, G. Giuffrida ${ }^{1}$, C. Sismeiro $^{2}$, and G. Tribulato ${ }^{1}$ \\ 1 University of Catania, Department of Mathematics and Computer Science, \\ Viale A. Doria 6, Catania, 95125, IT \\ \{battiato,gfarinella,ggiuffrida,tribulato\}@dmi.unict.it \\ 2 Imperial College London, Tanaka Business School, \\ South Kensington, Campus, London, SW7 2AZ, UK \\ sismeiro@imperial.ac.uk
}

\begin{abstract}
Traditionally, direct marketing companies have relied on pretesting to select the best offers to send to their audiences. Companies systematically dispatch the offers under consideration to a limited sample of potential buyers, rank them with respect to their performance and, based on this ranking, decide which offers to send to the wider population. Though this pre-testing process is simple and widely used, recently the direct marketing industry has been under increased pressure to further optimize learning, in particular when facing severe time and space constraints. Taking into account the multimedia nature of offers, which typically comprise both a visual and text component, we propose a two-phase learning strategy based on a cascade of regression methods. This proposed approach takes advantage of visual and text features to improve and accelerate the learning process. Experiments in the domain of a commercial Multimedia Messaging Service (MMS) show the effectiveness of the proposed methods that improve on classical learning techniques. The main contribution of the present work is to demonstrate that direct marketing firms can exploit the information on visual content to optimize the learning phase. The proposed methods can be used in any multimedia direct marketing domains in which offers are composed by image and text.
\end{abstract}

\section{Introduction and Motivations}

The importance of today's direct marketing industry is reflected by its significant economic value [1]. In recent years, as a result of technological advances in computing and communications, new channels for customer contact have become available, leading to the broadening of the direct marketing process. Beyond the traditional channels, which included traditional mail, catalog, and telephone contact, companies can now use multimedia channels as varied as email, mobile phone messaging, customized websites, addressable broadcasting, and directresponse TV and radio. It is this increase in the number of channels that has 
allowed, at least in part, the steady growth of direct marketing activities in recent years.

\subsection{The Multimedia Messaging Service Domain}

The domain of our empirical application is the targeting of multimedia messages (MMS) sent to mobile phone users, one of the new direct marketing channels. In this context a single multimedia message is sent everyday to the mobile phones of millions of customers. Each message contains a commercial offer and each offer advertises a specific product or service that can be purchased directly from the mobile phone with few clicks (e.g., a ringtone, a song, or a video).

From the mobile operator point of view this is in general very cost-effective: operators can easily reach millions of potential buyers at little cost, making the profit potential of these advertising-related services very high. In addition, given the mobile phone market saturation and fierce competition [2], value added services (VAS) and direct marketing offers are currently a significant revenue source for many mobile service providers. Because these services are now central to profitability, mobile phone operators are becoming increasingly creative in generating and proposing new services and offers to their customers. The result is a rapidly growing set of possible services available.

\subsection{Learning In Direct Marketing}

One of the challenges of direct marketing applications, like the one in our empirical domain, relates to the need of acquiring knowledge regarding the performance of all possible offers. Such knowledge is to be used in the development of better targeting and segmentation policies, which have always been at the core of the success of direct marketing activities [3,4].

Traditionally, direct marketing companies have relied heavily on pre-testing to acquire knowledge and select the best offers [5]. The pre-testing process is simple and widely used across the industry. First, the set of offers under consideration is sent to a limited sample of potential customers. Then, depending on the sample response, companies compare the performance of each offer and select the best offer for each segment of the population. Performance measures will vary depending on the specific application (catalogue, email, mobile messaging, etc.) and can include the number of items bought, the revenue per order, the click-through-rate (CTR), and the number of calls generated.

The best offers are the only ones sent to the wider population of potential customers, reducing waste (by avoiding sending irrelevant and potentially annoying messages to too many customers) and allowing higher performance and profitability. This specific phase may be accomplished by using response models approaches $[6,7]$.

The pre-testing process has worked well for the many direct marketing applications characterized by a low cost of contact and a large customer base (e.g., traditional mail). 


\subsection{The New Challenges of Direct Marketing Application}

The broadening of the direct marketing process and the creation of new channels, like the one in our empirical application, has brought new learning and knowledge acquisition challenges to the industry. First, the number of new offers in need to be tested in most of the new applications grows faster than the available opportunities for learning. Second, many of the offers have very short life-spans, posing additional pressure to produce fast learning. Under these circumstances the learning space may not be enough to learn on all available items.

For example, in the context of mobile messaging the number of alternative offers to be tested is extremely high and grows at a fast pace. It is not unusual to have more than 50,000 possible products or services to advertise at any moment, and the content catalogues can grow by twenty to thirty new items a day, a growth rate that is not likely to be reduced. In addition, even though there are millions of customers to contact, the number of opportunities for contact is small. Receiving too many commercial messages a day increases the likelihood that a customer will cancel a service or switch operator due to annoyance. Hence, operators do not want to fill customers' inboxes with too many messages. This means that one single person can only be exposed to no more than a very small fraction of all possible products and services. Hence, the limited size of mobile phone screens and the risk of alienating and overwhelming users if too many messages are sent daily, impose significant limits on the number of learning opportunities.

Given the speed of offer production, even with daily contact of customers (e.g., through messages sent to mobile phone users daily), the number of offers to be tested grows at a faster pace than the rate at which the system is able to learn while at the same time keeping enough potential customers for optimized delivery. Finally, offer expiration is now often close to its release date. In some cases offers need to be sent in a matter of days or even hours after they have been made available for selection and learning (e.g., news related services, holiday related offers). This further limits the opportunities and time for learning.

Hence, the rapid growth in the number of offers to be tested, the limited number of learning occasions, and the reduced time available for learning, make the traditional pre-testing not suitable in this context. This is because of significant time and learning space constraints: there are not enough opportunities for learning or, by the time enough learning could be gathered, the offers have already expired.

As a result, it is today essential for the mobile service providers to quickly understand the customer's needs and interests and select the right services to promote, at the right time, and in the right way. A good optimization and targeting system, that learns quickly and efficiently and that selects the right message/product to be sent to each customer so that revenues are maximized, could provide an additional profitability boost.

One significant advantage of mobile operators for this learning task is that the current infrastructure keeps detailed logs of all messages delivered and the response of each user. We can then track all messages and offers sent to customers 
(including their visual and text characteristics), and of the corresponding performance (e.g., whether the customer opened a message, viewed a page, bought a video, or clicked on a link). The information contained in these logs can then be used by an automated targeting system to aid message selection and customer targeting.

\subsection{The Present Work: Overview, Aims and Contributions}

This work is driven by the need of improving and accelerating the learning process in direct marketing applications under severe time and space constraints. We propose to optimize learning by taking advantage of the different features extracted from an offer's multimedia content (which include visual features).

The main contribution of this research is to demonstrate that one can make the learning phase more efficient and effective, with respect to the traditional learning phase, by taking advantage of the information on visual content together with the textual information present in each offer. The proposed strategy is assessed by simulation of an MMS direct marketing system using a real MMS dataset, and it outperforms traditional learning methods. Moreover, results demonstrate that the proposed learning strategy outperform the traditional learning also when the visual information are used alone.

We build on our initial work [8] in which the MMS "goodness" is derived combining text information with color based visual features. In that paper we showed that the combination of such simple color and text-related features performs better than when each type of feature is considered in isolation. Differently from [8] we propose here to capture the visual content of each offer's image as a whole entity through a holistic representation involving Textons [9-12]. Experiments presented in Section 5.3 show that a Textons-based representation achieves better results when compared against the representation used in [8]. In this paper the Textons-based representation of visual content for each MMS has been used together with text features for learning purpose.

Another contribution of this work is a new two-phase learning strategy in which a cascade of regressions methods is used to improve on classical learning methods. The suggested learning approach first learns through regression based methods which MMS offers available for learning should be subject to further testing, and which offers should wait for a better moment or simply be discarded. This first phase takes advantage of the static (non-behavioral) features extracted from image and text and from previous CTR information. In a subsequent phase, we propose to learn only on pre-screened MMS offers up to the limit of the system's learning capacity. This second-phase learning will take place just like in traditional direct marketing learning systems: the offers are sent to a sample of potential customers and the behavioral feedback registered and used for final offer selection. In our empirical application we show that the proposed approach can improve substantially the learning and optimization process, whenever direct marketing companies are operating under time and learning space constraints. 
The proposed learning method can be generalized and used in other multimedia direct marketing domains different from the MMS domain proposed in this paper.

The remainder of the paper is organized as follows: Section 2 introduces the dataset we have used to test our approach, the simulated MMS direct marketing system, and the main structure of our two-phase learning strategy. Section 3 describes the process we have employed to extract visual and text features from the MMS offers. In Section 4 we provide details on the regression tools, and on the cascade learning strategy that uses the extracted features. Section 5 reports the experiments and discusses the results in the MMS application domain. We conclude in Section 6 with avenues for further research.

\section{The Two-Phase Learning Approach for MMS Direct marketing}

The current section introduces the dataset and the basic concept used to simulate a real MMS Direct Marketing System. Moreover, the structure of the two-phase learning approach will be presented.

\subsection{The MMS Dataset}

To test the proposed approach, we use a real dataset of commercial multimedia messages sent to mobile users in Europe over a period of 15 months. There were more than one million users who opted-in for the service. There were also more than 70,000 possible direct marketing offers to advertise, but only a subset of 8600 items were labeled with a CTR due to the limit of our real system in sending more than twenty items per day.

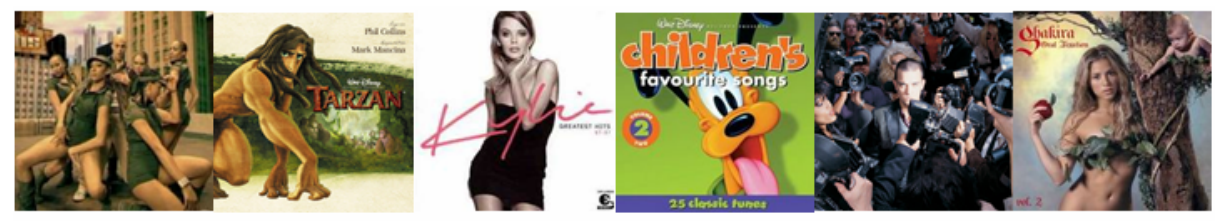

Fig. 1. Examples of available commercial MMS offers with resolution size $200 \times 200$.

Each commercial offer is composed by a small picture, a short description, and a price. The database we use contains the RGB thumbnail image associated with each offer. All images are encoded by JPEG standard with a high quality setting (i.e., no blocking is evident). The typical resolution size is $200 \times 200$ (see Figure 1) or $200 \times 116$ (see Figure 2). Our database includes also the short text description associated with each offer. This text message contains on average twelve words and briefly describes the commercial offer. Let's note that differently than [13] 
the text does not describe the image content. In particular the overall dataset contains several kinds of pictorial contents (e.g. face, people, building, cartoons, etc.) that is not directly related to the category and text of the offers. For each offer we also know the exact price charged to users who click on the offer and the click-through-rate of the offer across all mobile phone users.

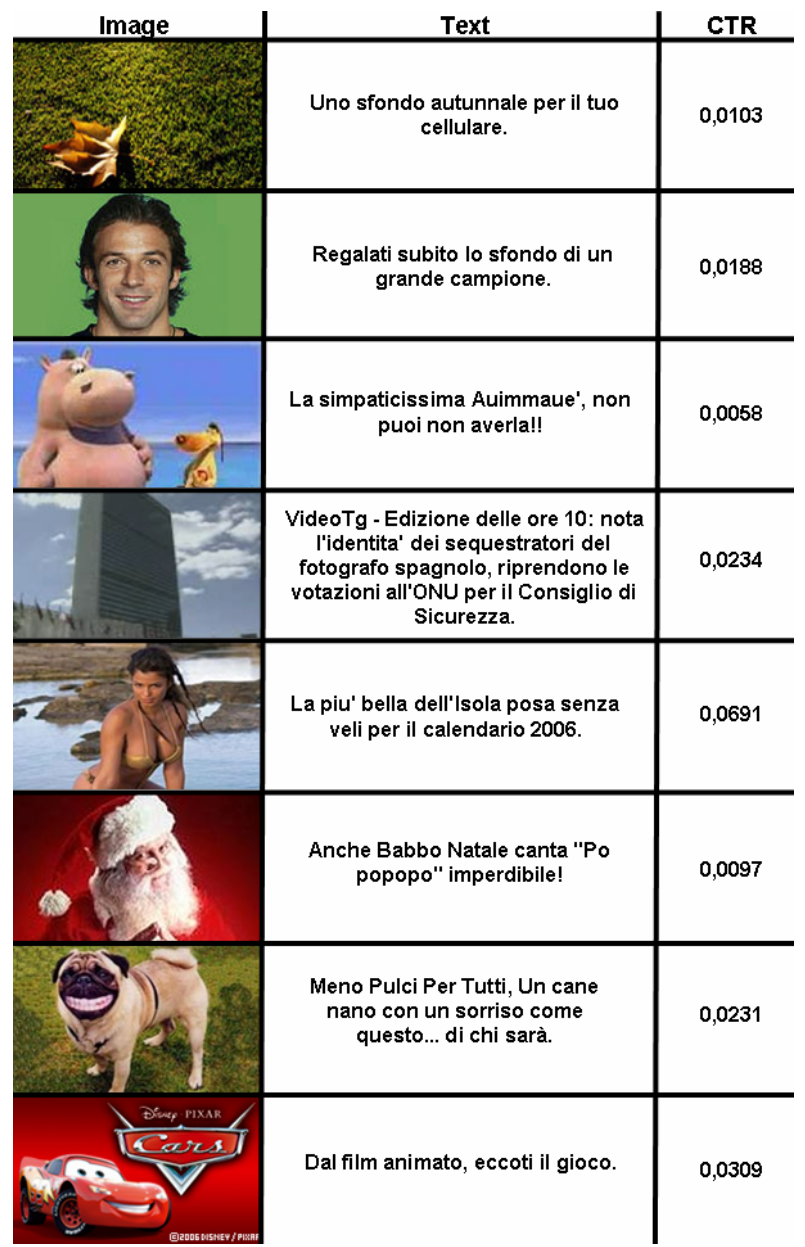

Fig. 2. Some examples of available commercial MMS offers with resolution size $200 \times 116$. An image and short text is associated to each offer. Moreover, the considered dataset is labeled with a real click-through-rate obtained sending the offers with a traditional method during a period of 15 months. 


\subsection{Simulating a Real MMS Direct Marketing System.}

Given the number of people in the customer base, and the reduced number of opportunities to contact them, we are able to learn only on a small number of new MMS offers each day. We define the sending rate to be the number of daily (learning) trials. This corresponds to the maximum number of MMS offers we can send to the learning panel each day (the learning panel being a set of customers we use for learning on new offers).

We define arrival rate as the average number of new MMS items added to the offer catalogue each day. We note that, before a final decision is made on which MMS items to send to the optimization portion of the customer base, we should learn on the revenue potential of each one of these new items.

As described in Section 1.3, in the type of applications we are considering, it is very likely that the arrival rate is greater than the sending rate. We call overcapacity rate the difference (arrival rate - sending rate). In addition, we call overcapacity the total number of unlearned messages. Hence, overcapacity measures the number of items on which we cannot learn, under the given learning space constraints. Since overcapacity grows monotonically each day (the rate of growth is given by the overcapacity rate), with a positive growth rate (common in the new direct marketing applications discussed previously) the learning task becomes more difficult over time.

Finally, we define base size as the number of offers that have never been sent to customers and are present at the beginning of the first learning phase (in a sense, the base size is the overcapacity measured just before the first learning phase takes place). If the overcapacity rate is greater than zero, the number of offers that have never been subject to learning increases over time (i.e., the overcapacity grows monotonically). This leads also to a growth of the base size just before a first learning phase is initiated.

\subsection{The Two-phase Learning Structure}

We propose a two-phase methodology to improve learning on new offers under time and learning space constraints. In Figure 3 the overall schema of our approach is reported.

We first estimate which of the offers available for learning are likely to be good (in terms of estimated CTR) by taking advantage of their static (nonbehavioral) visual and text features, and of the past CTR of similar (in terms of visual and text content) offers. As depicted in Figure 3, at the end of the first phase all new items will be labeled with the estimated CTR.

Then, in the second phase, we constrain learning to the offers with the highest performance potential until we reach the system's learning capacity. The performance potential of each offer is defined as the product between the estimated CTR and the price. This measure represents the expected revenue for each new delivered item. Learning in this phase takes place as in traditional direct market applications using the pre-testing approach discussed previously, that is, new offers are sent to the learning panel and the behavioral feedback (i.e., response) 


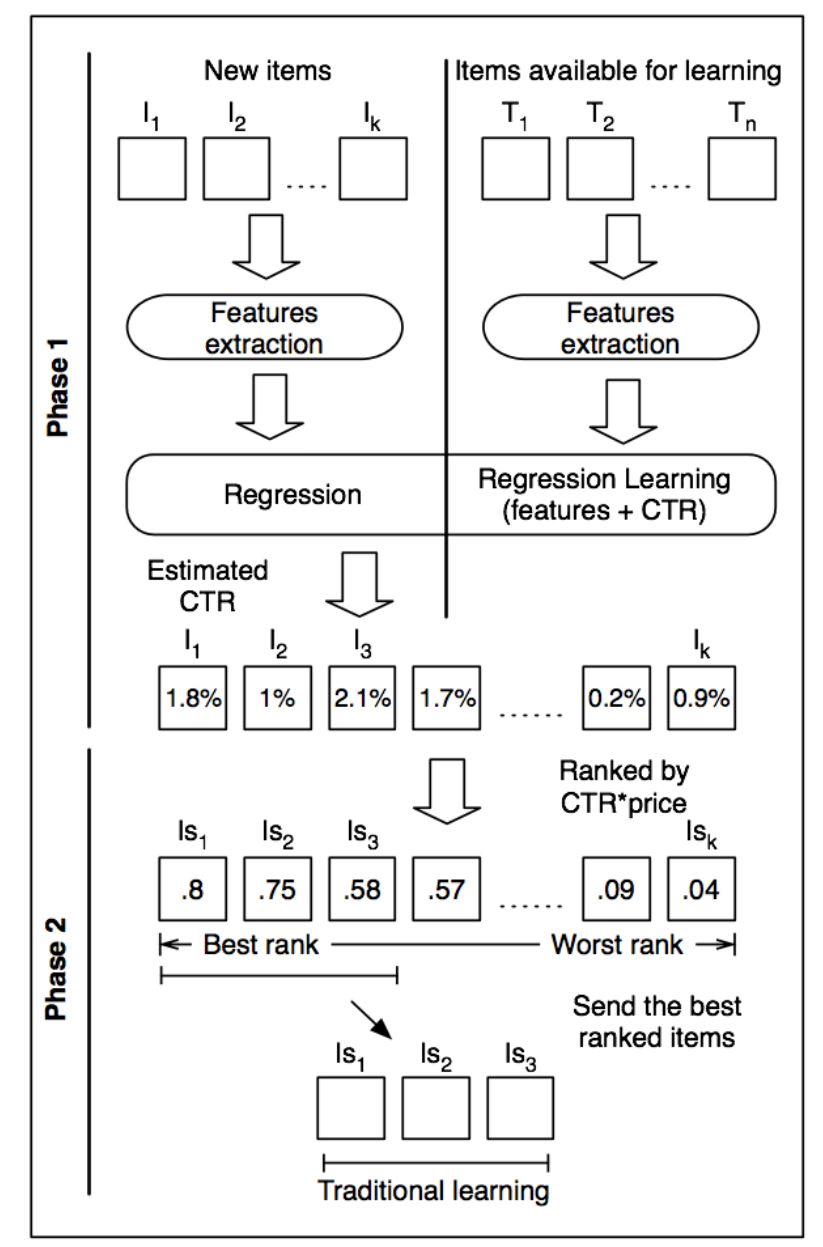

Fig. 3. The overall schema of the proposed two-phase learning approach.

is registered and used for final offer selection. In other words, we apply the traditional learning only to a subset of the newly arrived items. Such subset is chosen taking into account the performance potential of each item computed by using its static features. Thus, we limit the traditional learning to the most promising items for which we have enough time to learn on.

Results conduced by using the two-phase approach are discussed in Section 5 . We note that improving the learning procedure, by including the initial filtering (first phase learning), could potentially allow us to use a smaller sample of customers in the traditional learning phase and release customers for the more profitable optimized content delivery. 


\section{Exploiting Visual and Text features}

The first learning phase (See Section 2.3) uses visual and text features and the related known CTR of the commercial offers previously sent. Through automatic computer vision and text mining techniques we extract a set of static features that characterize the content of each MMS offer. The extracted features on past offers and the related effective CTR are used to train a regression-based method (See Section 4) in order to estimate the CTR for new items that will be used in the second learning phase.

\subsection{Holistic Encoding of Visual Content}

Customers' evaluation of the offer greatly depends upon their interpretation of the visual content. Thus, we use visual features to model how appeal an image offer can be for a user.

In [8] we proposed some colour based visual features to capture the main appearance of different objects and context. For instance, the presence of faces in an image can be inferred by the "skin appearance" in the pixel domain; likewise, an outdoor context can be inferred by "sky" and/or "vegetation" appearance [14]. Specifically, the following three types of visual information have been suggested to capture the appearance (see [8] for more details):

- Colors: full histograms and related statistics in the RGB, HSV, and CIE Lab color spaces;

- Filter Response: a complete set of band-pass filters to select both highfrequency details and global appearance;

- Semantic Analysis: to take into account objects and/or scene context we use pixel detectors using a Bayesian classifier for some involved object class (es. Sky, Skin, Vegetation).

These simple visual features, coupled with text features, revealed to be quite powerful for the learning phase, especially when compared to the performances obtained using only text characteristics [8].

Studies in Scene Perception and Visual Search [15-17] emphasize the fact that humans are able to recognize complex visual scenes at a single glance, despite the number of objects with different colors, shadows and textures that may be contained in the scenes. Moreover, recent works of Computer Vision research community [10,18-20] have efficiently exploited holistic cues $^{3}$ of an image to solve the problem of rapid and automatic scene classification bypassing the recognition of the objects inside the scene.

A holistic representation to capture the visual content of the scene as whole entity is a good deliverable in our application domain. More specifically, we have to choose a holistic representation able to capture the structure present in the MMS image.

${ }^{3}$ By definition, a holistic cue is one that is processed over the entire human visual field and does not require attention to analyze local features ([10]) 
As well known, humans can process texture quickly and in parallel over the visual field [21]: this makes texture a good candidate as holistic cue. The main process $[10,11]$ we used in this paper to encode textures cues starts by building a vocabulary of distinctive patterns, usually called visual words, able to identify properties and structures of different textures present in the offer's images. Using the built vocabulary each offer's image will be represented as an histogram of visual words.

To build the visual vocabulary each image in the training set is processed with a bank of filters. All pixel responses are then clustered pointing out a centroid for each cluster. These centroids, called Textons, represent the visual vocabulary. Each image pixels is then associated to the closest Textons taking into account its filter bank responses. Hence, each image becomes a "bag" of Textons (or visual words). A normalized histogram on Textons is used as descriptor for the holistic representation.

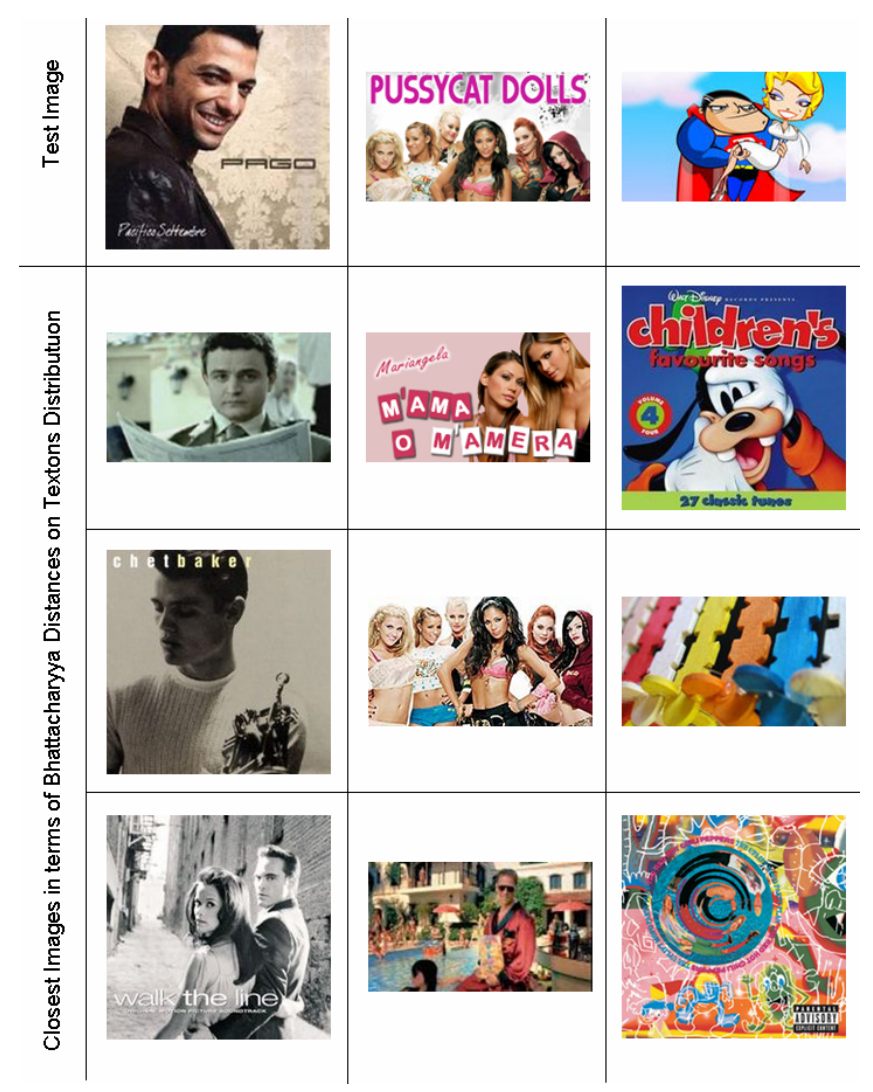

Fig. 4. Some test images are depicted at the top row. The closest training images in terms of Textons distribution are reported in the second and third row. The closest images are semantically consistent whit the related test images. 
In this work we use the bank of filters suggested in [12] and the k-means clustering algorithm to build the Textons vocabulary. More specifically, each pixel in each image has been associated with a 17-dimensional feature vector obtained by applying three Gaussian kernels $(\sigma=1,2,4)$, four Laplacian of Gaussian kernels $(\sigma=1,2,4,8)$ and 2 Derivative of Gaussian Kernels $(\sigma=2,4)$ on $\mathrm{x}$ and $\mathrm{y}$ directions. The Gaussian kernels have been applied on $L a b$ channels whereas the remaining filters only on $L$ channel.

In Figure 4 we report some images from the test dataset and the closest training images in terms of Textons distribution. The similarity between test and training images was computed by using a metric based on Bhattacharyya Coefficient [22] on Textons based representation discussed above. Note that there is a semantic consistence in contents between the test images and the corresponding closest training images.

\subsection{Extracting Features on Text Description}

To determine the static features of the short descriptive message of each offer, we collected the set of most common words among all messages in the training set (i.e., among all messages for which performance is assumed known).

Among all words extracted from the collection of messages we removed the least significant words and the stop words. The significance of a word is function of its entropy computed over the distribution of that word across all messages considered. That is, words that appear on a large percentage of messages tend to be less discriminant (thus, less significant) than words appearing on fewer messages. In addition, we removed also all words appearing in a very limited number of messages (less than five). This is justified by the low probability of finding the same word in the learning set, thus, it becomes very unlikely that we can use that word in the generalization phase.

After filtering out unlikely words, we dummified the learning space by creating a boolean variable for each word. Stemming [23] was considered but it doesn't improved the performances of the proposed approach. Thus, given a phrase, we set to one all Boolean variables corresponding to the words composing the text, and to zero otherwise. After processing the entire training set, we obtain a description of the text of each offer as a function of dummy variables. On average we extracted five words per message and used all of these in the first learning phase. Moreover, the TF-IDF normalization schema have been considered as text representation. Specifically, TF-IDF was employed for Locally Weighted Regression method in which an opportune metrics can be defined (see Next Section for more details).

\section{Learning on Different Feature Types by Cascade of Regression Methods}

As previously described, we obtain the static features for each MMS through automatic computer vision and text mining techniques (see Sections 3). Based on these features we create feature vectors for each MMS: 
- $f_{\text {Color }}$ : vector representing Colour based features to capture the appearance.

- $f_{\text {Textons }}$ : vector representing the probability distribution over the visual vocabulary (Textons) for each offer.

$-f_{T e x t}$ : vector representing the Text-based features for the short-text associated with each offer; the vector contains the dummy variables for the words present in the text.

Our objective is to predict the CTR for those items not yet sent to the population. Hence, the dependent variable of our learning problem is the CTR, which has been measured on items previously sent to mobile phone users (training set). For those messages not yet tested in the population, we use the predicted CTR to sort the offers and decide which ones to subject to further testing (i.e., to send to the second learning phase).

To perform the first learning phase and predict the CTR (see Section 2.3), we use the observed CTR and the static features of the commercial offers previously sent to users to train a regression-based model. Previous work has used in a similar context classification methods based on decision trees [8]. Because decision trees require the dependent variable to assume a discrete form, the authors in [8] convert CTR into performance classes (e.g., Good, Fair, Bad). Unlike this previous research, we propose that it is possible to perform a more accurate and robust learning process by taking into account the continuous nature of the CTR (our dependent variable). Hence, instead of decision trees, we propose to use regression-based methods that can better accommodate a continuous dependent variable.

We tested different regression models on our dataset: Regression Tree (RT) [24], Locally Weighted Regression (LWR) [25] and Support Vector Regression (SVR) [26]. We selected these regression methods based upon some properties of the involved features:

- Considering the binary nature of text features, RT could be a sensible choice. The use of RT also follows from what has been done in previous work [8] and allows us to test the performance of other regression-based methods against alternative models that rely on discrete dependent variables.

- Due to the complexity and non linearity of our problem (which involves image and text-based features), it is reasonable to look at the neighborhood of each feature vector to estimate the CTR of an offer. The LWR method allows exactly the use of localized information.

- The visual representation based on Textons encodes image as a whole entity; regression methods that take into account the entire representation of an offer, such as SVR, could then be more appropriate.

The RT method derives a set of if-then logical (split) conditions. This method does not assume any linear relationship between dependent and independent variables. One major drawback of RT models is that a threshold value determined in each stage of the tree construction does not correspond to the optimal boundary of bipartition of input subspace in that stage. To test the RT performances the WAGON library [27] has been used in our experiments. 
LWR is a memory-based method that performs a regression around a point of interest using only training data that are neighbors to that point. The basic assumption of LWR is that the neighborhood of each point in the dataset share similar values for the dependent variable. An important parameter in LWR is the metric to be used to compare similarity of features points. We note that, in using LWR, we have chosen a metric based on Bhattacharyya Coefficient [22] to compare Textons distribution whereas the L2 metric for Colour based representation. In addition, we employed the cosine similarity (dot product) and the Term Frequency Inverse Document Frequency (TFIDF) methodology [28] on Text features. The LWR algorithm was implemented by using [25] as reference.

SVR methods learn a nonlinear function in a kernel-induced features space. The parameters tuning and the choice of the best Kernel for the features space, remain the main disadvantage of this approach. In our experiments we have used the epsilon-SVR algorithm [29] within the LIBSVM library [30], and a radial basis function as kernel.

In Section 5 we will discuss experiments to discover which features $\left(f_{\text {Color }}\right.$, $f_{\text {Textons }}, f_{\text {Text }}$ ) leads to maximum performances. Moreover, we will point out that using only visual features our proposed methods outperform the traditional learning approach (see Section 5.4).

Considering the result obtained with the regression methods mentioned above (see Section 5 below) and taking into account the properties of the MMS feature representation (e.g., that Textons provide a global representation, that Text is represented by binary variables for each relevant word, etc.) we also tested a combination (also called cascade) of LWR and SVR methods as depicted in Figure 5 .

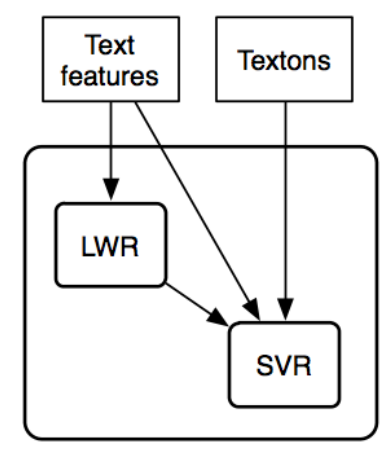

Fig. 5. The Cascade of Regression method involved in the first phase of our learning approach.

We believe the cascade of regression methods we propose is better suited to capture the properties of each static feature (Visual and Text) and takes into account the specific strengths of each regression method, as we will show below in our experimental results. 


\section{Experiments, Results and Discussion}

In this section, we describe in detail the experimental setup of our simulated MMS direct marketing system. Next we describe the results of our experiments using the proposed learning approach, and we then discuss our main findings.

\subsection{Experimental Setup}

We randomly split the dataset described in Section 2.1 into three partially overlapping subsets of 4500 MMS offers each (Figure 6). We sorted the MMS based on their arrival date and time and used the first $80 \%$ of the MMS offers

within each subset as training data. We retained the remaining $20 \%$ as our test data.

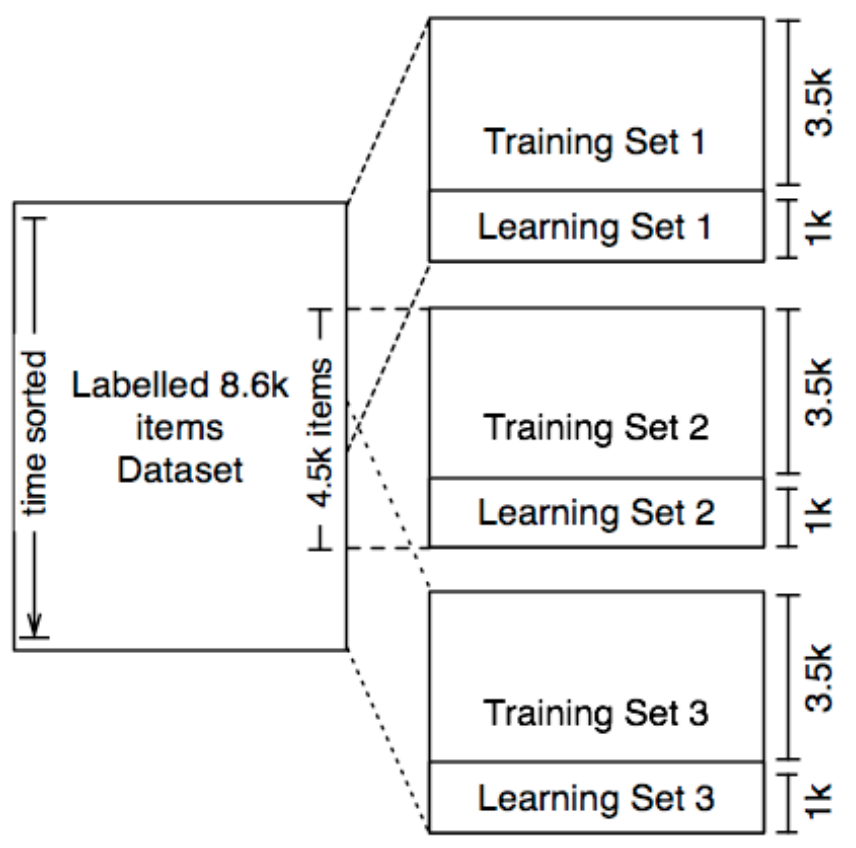

Fig. 6. The labeled dataset was subdivided in three subset to perform the testing phase. Each subset was used to simulate the MMS direct marketing system. The results relative to each subset were collected and used to analyze the performances of the proposed two phase learning approach.

We run the system simulation for each data subset and assume it works for 14 consecutive days. We set the sending rate, $S$, to 10 (the maximum number of daily trials) and varied the daily arrival rate $M(M=\{10,15,20,25, \ldots, 50,55\})$. For each value of the arrival rate, we assume it remains constant throughout 
the entire testing period. This means that, for each run, the overcapacity rate is constant and equal to $M-S$. Hence, each day, we accumulate $M-S$ new offers on which we are not able to learn. The initial offer catalogue size is zero (we start without any items requiring learning).

We apply the proposed two-phase approach to select the best offers to be sent for further testing (second learning phase, based on traditional learning), and determine the performance in the simulated system.

We test different visual vocabulary sizes (in the clustering of the visual features we tested for $\mathrm{k}=100,200,400$ where $\mathrm{k}$ is the number of clusters, i.e., the number of visual words). In the experiments presented in the following subsections, we used a vocabulary with 200 visual words because our preliminary tests revealed that this number provided the best results.

We tested for the number of dummy variables to use in representing the MMS text. We report on the experimental results based on the best number of dummy variables for each one of the methods applied. Specifically, through the filtering process discussed in Section 3.2, we obtain the best results using an average of about 1150 dummy variables in SVR and RT, and an average of 881 dummy variables in LWR.

The overall parameters used in our experiments are reported in Table 1.

\begin{tabular}{|c|c|c|}
\hline Regression Method & Parameter & Value \\
\hline \multirow{2}{*}{$\mathbf{L R}$} & Text Features & Binary Representation (1150) \\
\hline & Visual Features & Colour Based, Textons Based \\
\hline \multirow{3}{*}{ RT } & Text Features & Binary Representation (1150) \\
\hline & Visual Features & Colour Based, Textons Based \\
\hline & Stop $^{4}$ & 8 \\
\hline \multirow{8}{*}{ LWR } & Text Features & TF-IDF Representation (881) \\
\hline & Visual Features & Colour Based, Textons Based \\
\hline & Metric on Text & cosine similarity \\
\hline & Metric on Color & Euclidean $L_{2}$ \\
\hline & Metric on Textons & Bhattacharyya Coefficient \\
\hline & Polinomial Degree & 0 \\
\hline & NN-Bandwith & 39 \\
\hline & $K(d)=e^{-\frac{d}{\sigma}}$ & $\sigma=0.15$ \\
\hline \multirow{3}{*}{ SVR } & Text Features & Binary Representation (1150) \\
\hline & Visual Features & Colour Based, Textons Based \\
\hline & $K\left(x_{i}, x_{j}\right)=e^{-\gamma\left\|x_{i}-x_{j}\right\|^{2}}$ & $\gamma=\frac{1}{\# \text { features }}$ \\
\hline
\end{tabular}

Table 1. Regression Methods Settings. 


\subsection{System Evaluation}

As stated previously, for those messages not yet tested in the population, we use the predicted CTR (obtained during a first phase of learning) to sort the offers and decide which ones to subject to further testing (i.e., to send to a second phase of learning). Hence, we only perform additional tests to the most promising offers (up to system capacity). We assess each offer based on performance potential (see Section 2.3). We determine this potential by multiplying the estimated CTR of each MMS with its price (i.e., the revenue for the provider from each user click). Hence, once the learning phase is complete, we can compute the overall expected performance of any regression method (RM) taking into account the set of the chosen MMS offers as follows:

$$
\text { Performances }_{R M}=\sum_{m \in \Omega} C T R_{m} \times P R I C E_{m}
$$

where $C T R_{m}$ represents the real click-through-rate of the MMS $m \in \Omega$, and $P R I C E_{m}$ is the corresponding price.

We can also compute the overall system performances when we select randomly the offers to send to the population for testing (Performance $R A N D$ ) as in the traditional learning case. Note that Performance RAND represents the performance lower bound.

To assess the performance of the proposed approach, we use a data mining measure called Lift:

$$
\text { Lift }=\frac{\text { Performances }_{R M}}{\text { Performances }_{R A N D}}
$$

In this case, the Lift provides us a measure of how the proposed approach performs (in terms of revenue) relative to the traditional pre-testing (in which we do not pre-screen items for subsequent testing). In addition to the Lift measure, and to better assess the performances of the proposed learning approach in estimating the CTR of each offer, we also compute the root mean squared error $(R M S E)$ on the CTR predictions from the test data $\Psi$ :

$$
R M S E(\Psi)=\sqrt{\frac{\sum_{m \in \Psi}\left(\widehat{C T R}_{m}-C T R_{m}\right)^{2}}{|\Psi|}}
$$

RMSE is a frequently used measure of the the distance between the predicted values of a dependent variable and the true variable values for a given prediction method. Note also that in Equation 3 the CTRs values were normalized to the range $[0,1000]$.

The $R M S E(\Psi)$ and the Lift measures were computed for each simulation run. We then computed the average RMSE and Lift across all data subsets and across all runs. Below, we will report on such averages. 


\subsection{Comparison of Visual Features}

The colour based visual features we introduced so far (see Section 3.1) can be effectively exploited in estimating the CTR of an MMS offer. This statement is supported by the result obtained in [8] in which these features coupled with text features revealed their powerful during the learning phase. In that paper the experiments showed that adding visual information to text information achieves better performance compared to the text information when considered alone.

In this work we propose to use a holistic representation based on Textons that is more suitable to capture properties and structures of different textures inside the visual content of each MMS.

By using the $R M S E(\Psi)$ defined in previous section, we compare the two visual representations. Specifically, we are interested in understanding which representation achieve the best results in predicting the CTR of MMS offers. Moreover, we wish to understand which regression method (RT, LWR, SVR) gives the best result on visual representation and eventually why.

The $R M S E(\Psi)$ results useful for comparison are reported in Figure 7. As

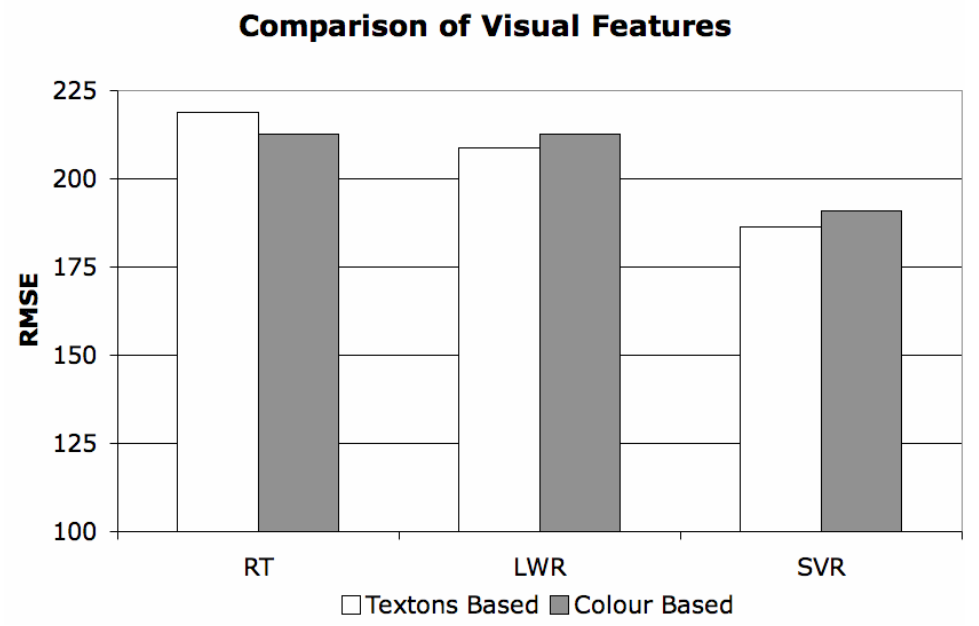

Fig. 7. Textons Based VS Color Based.

depicted in Figure $7, f_{\text {Textons }}$ features outperform $f_{\text {Colour }}$ features by using LWR or SVR. As expected the best results are obtained by using $f_{\text {Textons }}$ features and SVR. In fact SVR is able to properly work on the globally visual representation $f_{\text {Textons. }}$. The $f_{\text {Colour }}$ features heve reported better results by using RT because this regression method discriminate by looking at one single component feature at each level of the three. So RT is not able to treat properly the Textons based visual representation because it encode the MMS visual content as a whole entity by a global distribution. Anyway, SVR outperform RT independently by the 
involved features. These evidence allow us to assert that $f_{\text {Textons }}$ features and SVR are better suited for our application.

\subsection{Performances of Different Feature Types}

Because we can extract different types of features from our multimedia data (Textons Based and Text based), we first conduct a performance assessment when using each one of these features in isolation, with each one of the regression methods (RT, LWR, and SVR). To determine the most appropriate regression method for each type of feature when considered in isolation, we compare the $\operatorname{RMSE}(\Psi)$ of the each method-feature combination. This comparison will also allow us to select the best regression method we should use to extract knowledge from each feature (which we can then use when we combine features together in the regression cascade). Figure 8 reports the $R M S E(\Psi)$ results obtained using visual and text features when used in isolation when using the alternative regression models.

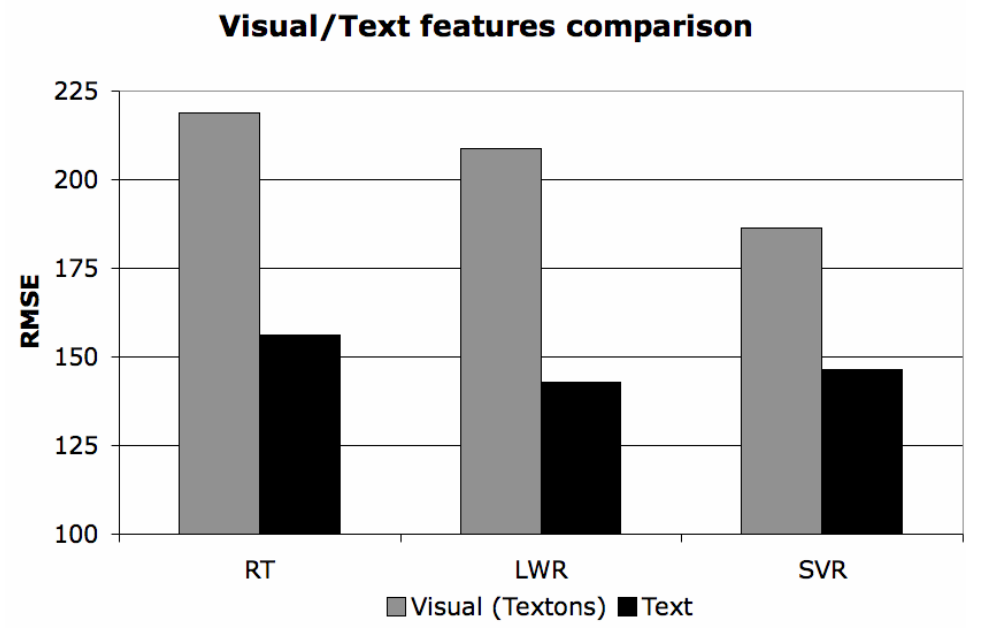

Fig. 8. $R M S E(\Psi)$ obtained with the three regression methods RT, LWR and SVR working on each kind of extracted features.

As we can see from Figure 8, for the Textons-based features we obtain the best results using the SVR model. We can conclude that the SVR is able to exploit the global visual representation of $f_{\text {Textons }}$, whereas other regression methods, like the RT, are not able to treat properly the Textons-based visual representation. These other methods rely on the information from individual components of the visual features at each level of the tree and do not consider the global information encoded by the combination of visual features of each MMS. 
We can make similar considerations for text-based features. RT is less powerful than the other two regression methods because it is not able to look at the word-related dummies jointly. However, to capture the semantic of an offer, it is better to take into account different words together than to look at each word singularly. LWR works well because the cosine similarity metric employed works well on the TF-IDF representation during the experiments.

As shown in Figure 8, we also find that text features perform better than visual features across all regression methods. One possible reason for this result is that the text might be working as a proxy for the offer's category (i.e., telling us whether the offers are connected to music, sports, phone wallpaper, etc.). In this specific domain application, we do not know a priori the offer's category (we do not have the classification of the offers into categories). Hence, we associate the power of text variables in predicting CTR for each MMS to the ability of the MMS short text in providing a category semantic. In contrast, the visual representation, though still showing predictive power, does not perform as well as the text. We conclude then that the visual component does not provide as clear clues as the text when predicting an offer's category or its likeability. Indeed, many of the offers from different categories are very similar in terms of visual content (Figure 9).

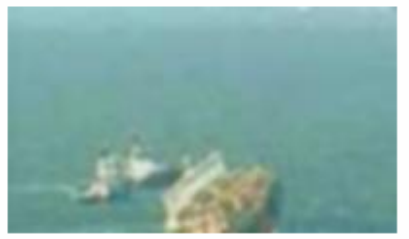

News

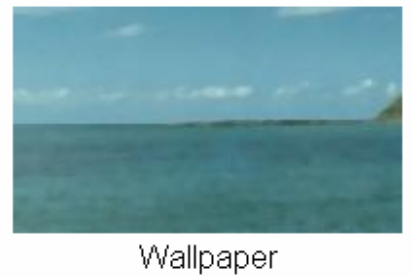

Wallpaper

Fig. 9. Two images extracted from our dataset. The images are similar in terms of visual content, but they come from two different category.

An important test we have done was devoted to understand if the two phase approach outperform the traditional just employing visual features. The Lift related the proposed approach by using SVR and visual features are reported in Figure 10.

As shown in Figure 10 our approach outperform the traditional learning also when visual features are used alone. The performances are better than traditional approach already at low arrival rate values and increase in a more constrained time and space domain. The improvement is more than $16 \%$ considering an arrival rate equal to 30 offers per day ${ }^{5}$. This means that in order to achieve better results, information on visual content can be effectively used in direct multimedia marketing application.

5 Taking into account the overall simulation settings, 30 offers per day is an arrival rate value comparable to the mean arrival rate values observed in our real system. 


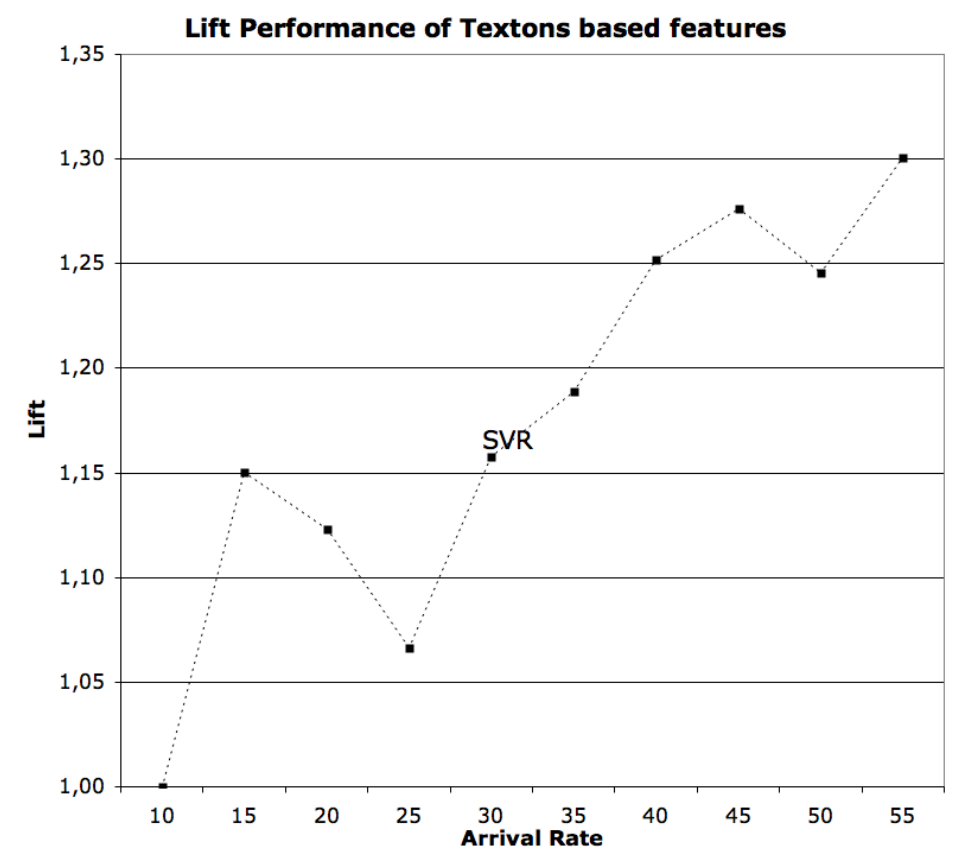

Fig. 10. The Lift results confirm that our approach outperform the traditional learning also using just visual features alone (e.g. Textons based).

In sum, one important result from our experiments is that text mining on an offers' short text might provide a good proxy for an offer's category, and as result, helps predict its CTR. This result suggests that combining the two sets of static features, visual and text-based, we are likely to obtain prediction improvements (as both sets of variables seem to capture different aspects of the message). This result further suggests that a greedy-like combination of regression methods could potentially provide additional improvements in CTR prediction. What we propose is to use a combination of regression methods known as a regression cascade. In this regression cascade, we first use text to infer the offers' category (using the best model for text features given by our previous analysis), and then we combine this result with the information that can be provided by visual features. We use visual features to find the most similar offers in terms of visual content, and again use the method most adequate for the visual features (as indicated by our initial experiments). We will describe in more detail the cascade of regression methods in next section.

Future works should be devoted in building a new visual representation by exploiting text first to have some prior about the offers' category [31]. 


\subsection{Exploiting Visual and Text features by Cascade of Regression Methods}

Taking into account the results of the previous sections, we have combined the different static features using the considered regression methods. We first combined the visual and text features using SVR model. This regression method obtained good performances on the two types of static features (see Figure 8). We report the $\operatorname{RMSE}(\Psi)$ results for the SVR model in Table 2. From Table 2 we

\begin{tabular}{|l|c|}
\hline Regression Approach & RMSE $(\Psi)$ \\
\hline $\operatorname{SVR}\left(f_{\text {Textons }}\right)$ & 186,48 \\
\hline $\operatorname{SVR}\left(f_{\text {Text }}\right)$ & 146,35 \\
\hline $\operatorname{SVR}\left(f_{\text {Text }}, f_{\text {Textons }}\right)$ & 145,83 \\
\hline
\end{tabular}

Table 2. The $R M S E(\Psi)$ results of using visual and text features singularly and jointly in SVR are reported. Combine together visual and text information give the best results.

can see that combining visual and text features provides better predictive accuracy, suggesting that both sets of variables indeed capture different aspects of the offers likeability (the SVR model using the visual and text features together outperforms the results obtained by when using each kind of feature separately). Hence, the Textons based features seem to be able to capture the visual content whereas, Text features add semantic information related the category of the offer. Despite these results, we note that SVR did not provide the best results for the text features (Figure 8). The best method to exploit text features is the LWR model. In addition, as discussed in the previous section, our findings seem to suggest that we should use text features before the visual component in order to capture the category of each MMS offer (e.g., music, sport, wallpaper, etc.). Then, we could add the visual features to discriminate between different visual content and improve predictive ability. This analysis induced us to use a greedy combination of features and regression methods. The final regression approach selected involved the LWR and the SVR models in cascade to take first advantage of the text features and then the visual features.

We tried two alternative formulations for the cascade of regressions. In the first one, we model the text features using the LWR. We then combined the CTR predicted using the LWR with the visual features, used a SVR model. The second alternative cascade tested included also text features directly in the SVR model in addition to the predicted CTR from the LWR output and the visual features (this cascade model is presented in Figure 5). We added text features again because the SVR and LWR models provided comparable result on these features, though they exploit the data in a very different manner (SVR looks at feature point in the global space whereas LWR looks locally around the features point). We then exploit again the text features directly in the SVR to 
take advantage of the different types of information contained in the text. We report the $R M S E(\Psi)$ results of these cascade regressions in Table 3.

\begin{tabular}{|l|c|}
\hline Regression Approach & RMSE $(\Psi)$ \\
\hline $\operatorname{SVR}\left(f_{\text {Text }}, f_{\text {Textons }}\right)$ & 145,83 \\
\hline $\operatorname{SVR}\left(\operatorname{LWR}\left(f_{\text {Text }}\right), f_{\text {Textons }}\right)$ & 144,04 \\
\hline $\operatorname{SVR}\left(f_{\text {Text }}, \operatorname{LWR}\left(f_{\text {Text }}\right), f_{\text {Textons }}\right)$ & 142,82 \\
\hline
\end{tabular}

Table 3. The RMSE $(\Psi)$ results of using visual and text features in SVR are reported in comparison with the results obtained by the proposed cascade approach. A cascade of regression methods achieve best results.

From the analysis of Table 3 we conclude that the regression cascade improves the predictive ability of our system, estimating more accurately the CTR of each offer. The results seem also to suggest that this method can better capture the properties of each kind of representation (Visual, Text), and take into account of the peculiarity of each regression method.

To better understand the performances of the overall system using the cascade approach, we computed the Lift for alternative model formulations. In Figure 11, we present these Lift results. It is clear from the figure that using the proposed approach (irrespective of the final regression formulation) improves significantly the overall performance of the system producing significantly higher revenue. This is because we selected the best offers during the first learning phase to be subject to further learning, whereas we discarded the weakest options did not wasted the scarce testing opportunities with offers that a priori seemed too weak.

From Figure 11 we can see that the direct marketing system outperforms the traditional learning approach in more than $35 \%$ when the arrival rate is 30 items per day (a value comparable to the one in the real system) that is comparable to the mean arrival rate value observed in our real system. In addition, we can clearly see that the performances of the system increases as the system constraints become more severe (more than 30 items arrival per day) reaching $45 \%$ of improvement when compared to the traditional testing system. It is interesting to note also that the proposed approach outperform the traditional learning approach also when using only visual features. In such case, the proposed approach outperforms the traditional testing in more than $16 \%$ when the arrival rate is 30 offers per day.

\section{Conclusion and Future Works}

A successful approach to improve the performance of direct marketing systems on new multimedia channels, will require the contribution of wide range of disciplines and technologies including computer vision, data mining, statistics and 


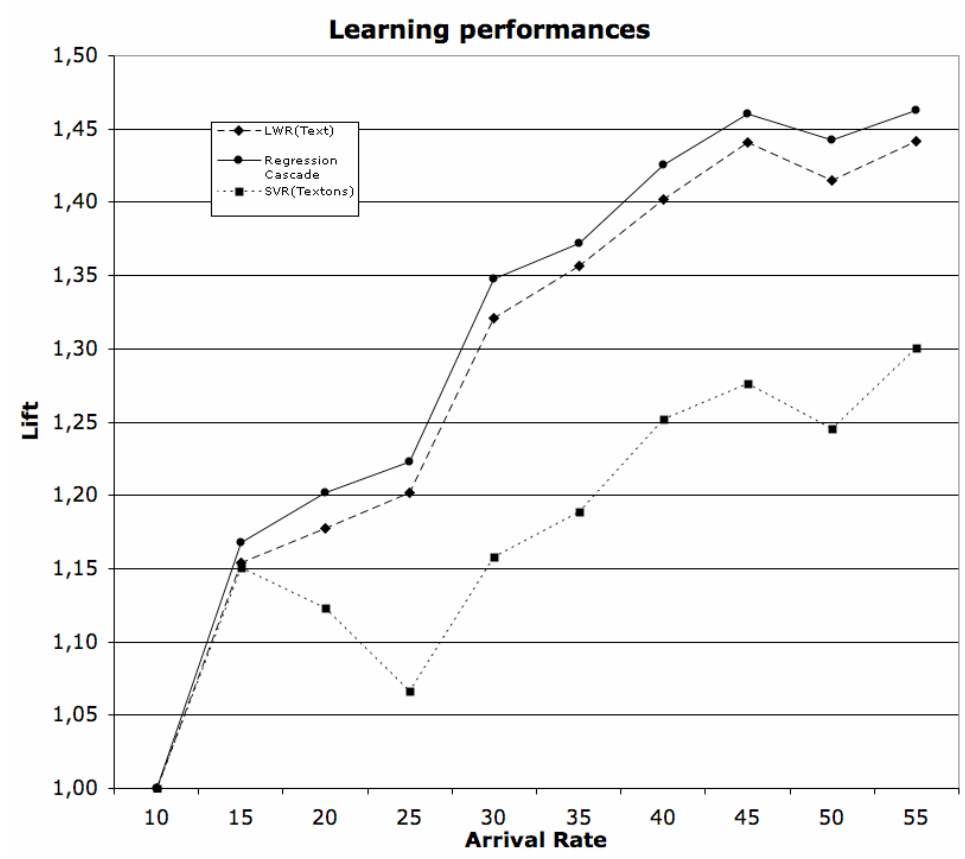

Fig. 11. The plot reports the Lift result obtained by using visual and text features singularly and by using the cascate of regressiom method.

marketing. In this paper, we presented an application domain where we learning needs to take place under severe time and space constraints. In particular, we propose a two-phase learning approach for a direct marketing application that relies on Multimedia Messaging Service (MMS) for offer delivery. In our approach, we propose to exploit the visual and text features of each MMS offer through a cascade of regression algorithms to estimate the potential (in terms of expected revenue) of each offer. We demonstrated that this approach leads to a considerable improvement in overall performance. Researchers and businesses can also use the presented schema in other domains in which visual and text information is available (e.g. offers sent by email, interactive television, etc).

Moreover, the results obtained during experimental phase have shown good performances also when visual features are used alone. This means that when visual information (e.g. images) are present, exploit information on visual content can make more efficient and effective the learning phase.

Future work would require the evaluation of online techniques for the learning phase strategy (e.g. online boosting [32]), and could take into account also other active learning strategies based on uncertain sampling. The discriminant power of other visual features should be evaluated. In addition, customers' behavior and the offer's price could be exploited as features and be used jointly with visual and text features to predict performance. Finally, the combination of different 
types of features able to capture text and visual contents should be tested also in other direct marketing domains.

\section{Acknowledgments}

The authors would like to thank Daniele Ravì for helping in the implementation of the simulation studies. The authors would also like to thank Neodata Group for giving access to the mobile messaging dataset, and for helping in the implementation and testing of the proposed approach.

\section{References}

1. Direct Marketing Association: The Power of Direct Marketing: ROI, Sales, Expenditures and Employment in the U.S., 2006-2007 Edition. Direct Marketing Association (2007)

2. Netsize: Convergence: Everything is going mobile. The Netsize Guide 2007. Netsize (2007)

3. Roberts, M., Berger, P.: Direct Marketing Management. Prentice-Hall (1989)

4. Prinzie, A., Van Den Poel, D.: Constrained optimization of data-mining problems to improve model performance: A direct-marketing application. Expert Systems with Applications 29(3) (2005) 630-640

5. Nash, E.: Direct Marketing. McFraw-Hill (2000)

6. Cui, G., Wong, M.L., Lui, H.K.: Machine learning for direct marketing response models: Bayesian networks with evolutionary programming. Manage. Sci. 52(4) (2006) 597-612

7. Baesens, B., Viaene, S., Van den Poel, D., Vanthienen, J., Dedene, G.: Bayesian neural network learning for repeat purchase modelling in direct marketing. European Journal of Operational Research 127(1) (2002) 191-211

8. Battiato, S., Farinella, G., Giuffrida, G., Tribulato, G.: Data mining learning bootstrap through semantic thumbnail analysis. In: SPIE-IS\&T 19th Annual Symposium Electronic Imaging Science and Technology 2007 - Multimedia Content Access: Algorithms and Systems. (2007)

9. Julesz, B.: Textons, the elements of texture perception, and their interactions. Nature 290 (1981) 9197

10. Renninger, L.W., Malik, J.: When is scene recognition just texture recognition? Vision Research 44 (2004) 2301-2311

11. Varma, M., Zisserman, A.: A statistical approach to texture classification from single images. Int. J. Computer Vision 62(1-2) (2005) 61-81

12. Winn, J., Criminisi, A., Minka, T.: Object categorization by learned universal visual dictionary. In: ICCV '05: Proceedings of the Tenth IEEE International Conference on Computer Vision, Washington, DC, USA, IEEE Computer Society (2005) 1800-1807

13. Barnard, K., Forsyth, D.A.: Learning the semantics of words and pictures. In: ICCV. (2001) 408-415

14. Naccari, F., Battiato, S., Bruna, A., Capra, A., Castorina, A.: Natural scene classification for color enhancement. IEEE Transactions on Consumer Electronics 5 (2005) 234-239 
15. Biederman, I., Mezzanotte, R., Rabinowitz, J.: Scene perception: Detecting and judging objects undergoing relational violations. Volume 14. (1982) 143-177

16. Biederman, I.: Recognition by components: Atheory of human image interpretation. Psychological Review 94 (1987) 115-148

17. Potter, M.: Meaning in visual search. Science 187 (1975) 965-966

18. Oliva, A., Torralba, A.: Modeling the shape of the scene: a holistic representation of the spatial envelope. International Journal of Computer Vision 42 (2001) 145-175

19. Fei-Fei, L., Perona, P.: A bayesian hierarchical model for learning natural scene categories. In: IEEE Conference on Computer Vision and Pattern Recognition. (2005)

20. Lazebnik, S., Schmid, C., Ponce, J.: Beyond bags of features: Spatial pyramid matching for recognizing natural scene categories. In: IEEE Conference on Computer Vision and Pattern Recognition. Volume II. (2006) 2169-2178

21. Bergen, J.R., Julesz, B.: Rapid discrimination of visual patterns. IEEE Transactions on Systems, Man, and Cybernetics 13 (1983) 857-863

22. Comaniciu, D., Ramesh, V., Meer, P.: Kernel-based object tracking. IEEE Trans. Pattern Anal. Mach. Intell. 25(5) (2003) 564-575

23. Hull, D.: Stemming algorithms: a case study for detailed evaluation. Journal of the American Society for Information Science 47 (1996) 70-84

24. Breiman, L., Friedman, J., Olshen, R., Stone, C.: Classification and Regression Trees. Wadsworth and Brooks, Monterey, CA (1984)

25. Cleveland, W.S., Devlin, S.J., Grosse, E.: Regression by local fitting : Methods, properties, and computational algorithms. Journal of Econometrics 37(1) (1988) 87-114

26. Shawe-Taylor, J., Cristianini, N.: Support Vector Machines and other kernel-based learning methods. Cambridge University Press (2000)

27. Taylor, P., Caley, R., Black, A.W., King, S.: Wagon, edinburgh speech tools library (1994-1999)

28. Oren, N.: Reexamining tf.idf based information retrieval with genetic programming. In: SAICSIT '02: Proceedings of the 2002 annual research conference of the South African institute of computer scientists and information technologists on Enablement through technology, , Republic of South Africa, South African Institute for Computer Scientists and Information Technologists (2002) 224-234

29. Schölkopf, B., Smola, A.J., Williamson, R.C., Bartlett, P.L.: New support vector algorithms. Neural Comput. 12(5) (2000) 1207-1245

30. Chang, C.C., Lin, C.J.: LIBSVM: a library for support vector machines. (2001)

31. Florent, P.: Universal and adapted vocabularies for generic visual categorization. Pattern Analysis and Machine Intelligence, IEEE Transactions on : Accepted for future publication (2007)

32. Oza, N.C.: Online bagging and boosting. In: Systems, Man and Cybernetics, 2005 IEEE International Conference on. (2005) 2340-2345 